\title{
N89-20336
}

\section{2-GHz Cryogenically Cooled HEMT Low-Noise Amplifiers}

\author{
J. J. Bautista and G. G. Ortiz \\ Radio Frequency and Microwave Subsystems Section \\ K. H. G. Duh, W. F. Kopp, P. Ho, P. C. Chao, M. Y. Kao, \\ P. M. Smith, and J. M. Ballingall \\ GE Electronics Laboratory
}

\begin{abstract}
The cryogenic noise temperature performance of a two-stage and a three-stage 32-GHz HEMT amplifier has been evaluated. The amplifiers employ 0.25- $\mu$ m conventional AlGaAs/ GaAs HEMT devices, hybrid matching input and output microstrip circuits, and a cryogenically stable dc biasing network. The noise temperature measurements were performed in the frequency range of 31 to $33 \mathrm{GHz}$ over a physical temperature range of $300 \mathrm{~K}$ down to $12 \mathrm{~K}$. Across the measurement band, the amplifiers displayed a broadband response, and the noise temperature was observed to decrease by a factor of 10 in cooling from $300 \mathrm{~K}$ to $15 \mathrm{~K}$. The lowest noise temperature measured for the two-stage amplifier at $32 \mathrm{GHz}$ was $35 \mathrm{~K}$ with an associated gain of $16.5 \mathrm{~dB}$, while the three-stage amplifier measured $39 \mathrm{~K}$ with an associated gain of $26 \mathrm{~dB}$. It was further observed that both amplifiers were insensitive to light.
\end{abstract}

\section{Introduction}

Traditionally, the extraordinarily sensitive receiver systems operated by the Jet Propulsion Laboratory's Deep Space Network (DSN) have employed ruby masers as the low-noise front-end amplifiers. The rapid advances recently achieved by cryogenically cooled high-electron-mobility transistor (HEMT) low-noise amplifiers (LNAs) in the 1- to $10-\mathrm{GHz}$ range are approaching maser amplifier performance [1], [2]. In order to address its future spacecraft navigation, telemetry, radar, and radio science needs, the DSN is investigating both maser [3] and HEMT amplifiers for its $32-\mathrm{GHz}$ downlink capability. This report describes the noise temperature performance of the $32-\mathrm{GHz}$ HEMT LNAs.

\section{HEMT Device}

Since one of the primary functions of the LNA is to minimize the receiver system noise temperature, the characterization and selection of HEMT devices is critical to the LNA's performance. The selection of the $0.25-\mu \mathrm{m}$ gate length conventional AlGaAs/GaAs HEMTs was based on their previously demonstrated reliability and exceptionally high gain and low noise characteristics [4], [5].

The devices were fabricated on selectively doped AlGaAs/ GaAs heterostructures grown by molecular beam epitaxy (MBE) with a Varian GEN II system on a 3-inch-diameter GaAs substrate. The details of the material growth conditions 
are discussed elsewhere [6]. Figure 1 schematically illustrates the cross section of the HEMT device. The HEMT wafer exhibited a sheet carrier density of $8.1 \times 10^{12} / \mathrm{cm}^{2}$ with a mobility of more than $75,000 \mathrm{~cm}^{2} / \mathrm{V} \cdot \mathrm{sec}$ at $77 \mathrm{~K}$. All levels were defined by electron beam lithography, and the T-shape gates were fabricated using the PMMA/P(MMA-MMA)/PMMA tri-layer resist technique [7] to achieve a low series gate resistance.

For low-noise performance at cryogenic temperatures, the HEMT device must exhibit good pinch-off characteristics and high transconductance, $g_{m}$. Good pinch-off characteristics are achieved by strong confinement of the charge carriers to the channel region with a sharp interface of high quality and a large conduction band discontinuity. An enhanced $g_{m}$ at the operating bias is obtained by a judicious choice of doping concentration and space layer thickness [8]. An Al mole fraction of approximately $30 \%$ is required for a large conduction band discontinuity, while the high $\mathrm{g}_{\mathrm{m}}$ is achieved with a 4-nm spacer layer and a doping concentration of approximately $2 \times 10^{18}$ dopant atoms $/ \mathrm{cm}^{3}$. Although these values will result in a high-performance room-temperature device, at physical temperatures below $150 \mathrm{~K}$ the device will suffer from I-V collapse [9] and exhibit the persistent photoconductivity effect associated with the presence of deep donor traps (called DX centers). In order to obtain excellent device performance at cryogenic temperatures and to eliminate light sensitivity, previous work [1], [8] has demonstrated that the Al composition must not exceed $23 \%$ and the doping concentration must be approximately $10^{18} / \mathrm{cm}^{3}$.

The data shown in Table 1, comparing two HEMTs with the same Al mole fraction (23\%) but different doping concentrations in the n-AlGaAs layer, serves to illustrate the difference between low-temperature and room-temperature device optimization. Device $A$ has an $\mathrm{n}-\mathrm{AlGaAs}$ doping concentration of $10^{18} / \mathrm{cm}^{3}$, while that of $B$ is twice as high. As expected, device $B$ exhibited a higher $g_{m}$ and associated gain than device $A$, with approximately the same noise figure for both devices at $300 \mathrm{~K}$. However, at $13 \mathrm{~K}$ and $8.5 \mathrm{GHz}$, device B exhibited a minimum noise temperature of $13.1 \mathrm{~K}$, while device $\mathrm{A}$ yielded a value of $5.3 \mathrm{~K}$.

\section{Amplifier Design and Circuit}

Both LNAs were designed to achieve the best room-temperature low-noise performance based on the measured roomtemperature device parameters. Following construction and room-temperature optimization, the LNAs are then biased for lowest noise performance at cryogenic temperatures.

The device gate width of $75 \mu \mathrm{m}$ selected for this work was determined by the tradeoffs associated with optimum impe. dance matching, circuit bandwidth, intermodulation distortion, power handling capability, and power dissipation. Figure 2 shows a photograph of the 32-GHz hybrid two-stage HEMT LNA package. The input and output ports utilize a broadband WR28-to-stepped ridge waveguide-to-microstrip transition. Figure 3 shows the insertion loss and return loss of a stepped ridge fixture that consists of two stepped-ridge transitions connected back-to-back with a microstrip 50 -ohm line 0.5 in. long. The input and output matching networks were designed based on the device equivalent circuit values obtained from fitting measured S-parameters at the low-noise bias condition to the model from 2 to $20 \mathrm{GHz}$. Figure 4 shows the topology used for the $0.25-\mu \mathrm{m}$ HEMT equivalent circuit model. Input, output, and interstage matching circuits were designed on 10-mil quartz substrate with TaN thin-film resistors and TiWAu metallization. A schematic diagram of the two-stage hybrid HEMT LNA is shown in Fig. 5. The edge-coupled symmetric microstrip dc blocking transmission line also served as a bandpass filter, improving the out-of-band stability. As shown in Fig. 6, the three-stage LNA is constructed from the two-stage LNA by the insertion of another interstage matching circuit.

The LNA fixture (OFHC copper) and dc bias circuits [10] are designed for operation at cryogenic temperatures. Diode protection was included in both the gate and drain bias circuits. LEDs were mounted on the cover of the fixture above each of the HEMTs for the purpose of examining their light sensitivity at cryogenic temperatures. All of the stages use devices from the same wafer.

\section{Measurement Results}

The LNAs were first measured at room temperature with the devices biased for lowest noise at room temperature and then biased for lowest noise performance at cryogenic temperatures. The LNA room-temperature broadband noise figure and gain for the two- and three-stage LNAs are shown in Figs. 7 and 8 , respectively. Both LNAs exhibited an average noise figure of approximately $2 \mathrm{~dB}$ from 28 to $36 \mathrm{GHz}$. From 29 to $34 \mathrm{GHz}$, the gain measured approximately $17 \mathrm{~dB}$ and $24 \mathrm{~dB}$ for the two-stage and three-stage LNA, respectively. The addition of an external isolator only slightly degraded the gain and noise figure by $0.3 \mathrm{~dB}$.

With the devices biased for lowest noise at cryogenic temperature $(12 \mathrm{~K})$, the noise temperature (referenced at the room-temperature input waveguide flange) of both LNAs was observed to decrease nearly quadratically as a function of physical temperature as they cooled from $300 \mathrm{~K}$ to $12 \mathrm{~K}$. (See Fig. 9 for a diagram of the closed-cycle refrigerator and measurement system.) The noise temperature of the twostage LNA decreased from $350 \mathrm{~K}$ at ambient to $35 \mathrm{~K}$ at 
$14.5 \mathrm{~K}$, while the three-stage LNA decreased from $400 \mathrm{~K}$ to $41 \mathrm{~K}$ at $12.5 \mathrm{~K}$ (Figs. 10 and 11 ). Figures 12 and 13 show the cryogenic noise temperature and gain response from 31 to $33 \mathrm{GHz}$, along with bias settings for the two-stage and threestage LNA, respectively. At $32 \mathrm{GHz}$, the two-stage LNA noise temperature measured $35 \mathrm{~K}$, with an associated gain of $16.5 \mathrm{~dB}$, at a physical temperature of $14 \mathrm{~K}$, while the three-stage LNA yielded a value of $41 \mathrm{~K}$ with a $26.0-\mathrm{dB}$ associated gain. It is also noted that the three-stage LNA displayed an almost flat noise temperature response across the measurement band, with a minimum noise temperature of $39 \mathrm{~K}$ at $32 \mathrm{GHz}$, while the two-stage LNA displayed a noise temperature response decreasing monotonically from 31 to $33 \mathrm{GHz}$, with a minimum noise temperature of $31 \mathrm{~K}$ at $33 \mathrm{GHz}$.

It was further observed that both amplifiers did not show a persistent photoconductivity effect. That is, it was found that these devices can be cooled with or without illumination and/or dc bias, without any observable effect on the cryogenic low-noise performance.

\section{Conclusion}

Cryogenic coolable state-of-the-art 32-GHz HEMT LNAs have been demonstrated using $0.25-\mu \mathrm{m}$ AlGaAs/GaAs HEMTs. The results clearly demonstrate their potential to meet the future need for extremely low-noise receivers for applications such as the DSN. Further advances in HEMT technology [12] promise to lead to improved performance at all frequencies and make possible the development of amplifiers operating at frequencies up to $94 \mathrm{GHz}$.

Currently, the DSN relies on maser amplifiers in order to provide the best possible telemetry support for deep space missions. These systems require a complex and expensive cryogenic system operating at $4.5 \mathrm{~K}$. Since HEMT LNAs require less cooling power and operate at a higher physical temperature $(12 \mathrm{~K})$, they can be operated at less cost with a more reliable refrigeration system. The lower cost of HEMT LNAs will lead to greater frequency coverage and the economic realization of multiple-element cryogenic array feed systems.

\section{Acknowledgments}

The authors wish to thank M. Pospieszalski for providing X-band cryogenic HEMT data, and J. Merrill for the waveguide transition design. The authors would also like to acknowledge the support of A. A. Jabra, D. Neff, J. Bowen, and D. Norris. GE Electronics Laboratory's 32-GHz cryogenic low-noise HEMT development was supported by JPL under Contract No. 957352. 


\section{References}

[1] M. W. Pospieszalski, S. Weinreb, P.C.Chao, U.K. Mishra, S.C. Palmateer, P. M. Smith, and J. C. M. Hwang, "Noise Parameters and Light Sensitivity of Low-Noise HighElectron-Mobility Transistors," IEEE Trans. Electron Devices, vol. ED-33, pp. 218 $223,1986$.

[2] S. Weinreb, M. W. Pospieszalski, and R. Norrod, "Cryogenic, HEMT, Low-Noise Receivers for 1.3 to $.43 \mathrm{GHz}$ Range," IEEE MTT S Digest, pp. 945-948, 1988.

[3] J. Shell and D. Neff, "A 32-GHz Reflected Wave Maser Amplifier with Wide In. stantaneous Bandwidth," IEEE MTT-S Digest, pp. 789-792, 1988.

[4] K. H. G. Duh, P. C. Chao, P. M. Smith, L. F. Lester, B. R. Lee, J. M. Ballingall, and M. Y Kao, "Millimeter-Wave Low-Noise HEMT Amplifiers," IEEE MTT-S Digest, pp. 923-926, 1988.

[5] P. M. Smith, P. C. Chao, K. H. G. Duh, L. F. Lester, B. R. Lee, J. M. Ballingall, and M. Y. Kao, "Advances in HEMT Technology and Applications," IEEE MTT-S Digest, pp. 749-752, 1987.

[6] S. C. Palmateer, P. A. Maki, W. Katz, A. R. Calawa, J.C. M. Hwang, and L. F. Eastman, "The influence of V:III flux ratio on unintentional impurity incorporation during molecular beam epitaxial growth," in Proc. Gallium Arsenide and Related Compounds 1984 (Inst. Phys. Conf. Series 74), pp. 217-222, 1985.

[7] P. C. Chao, P. M. Smith, S. C. Palmateer, and J. C. M. Hwang, "Electron-Beam Fabrication of GaAs Low-Noise MESFETs Using a New Tri-Layer Resist Technique," IEEE Trans. Electron Devices, vol. ED-22, pp. 1042-1046, 1985.

[8] K. H. G. Duh, M. W. Pospieszalski, W. F. Kopp, P. Ho, A. A. Jabra, P. C. Chao, P. M. Smith, L. F. Lester, J. M. Ballingall, and S. Weinreb, "Ultra-Low-Noise Cryogenic High-Electron Mobility Transistors," IEEE Trans. Electron Devices, vol. ED-35, pp. 249-256, 1988.

[9] A. Kastarsky and R. A. Klein, "On the low temperature degradation of AlGaAs/ GaAs modulation doped field-effect transistors," IEEE Trans. Electron Devices, vol. ED-33, pp. 414-423, 1986.

[10] S. Weinreb and R. Harris, "A 23-GHz Coolable FET Amplifier," NRAO Internal Report.

[11] P. C. Chao, P. M. Smith, K. H. G. Duh, J. M. Ballingall, L. F. Lester, B. R. Lee, A. A. Jabra, and R. C. Tiberio, "High Performance $0.1 \mu \mathrm{m}$ Gate-Length PlanarDoped HEMTs," 1987 International Electron Devices Meeting, Washington, D. C., Dec. 1987, Paper 17.1, pp. 410-413.

[12] P. Ho, P. C. Chao, K. H. G. Duh, A. A. Jabra, J. M. Ballingall, and P. M. Smith, "Extremely High Gain, Low Noise InAlAs/InGaAs HEMTs Grown by Molecular Beam Epitaxy," to be published in 1988 IEDM Technical Digest. 
Table 1. Performance comparison of two types of conventional AIGaAs/GaAs HEMTs

\begin{tabular}{cclcc}
\hline $\begin{array}{c}\text { Ambient } \\
\text { Temp., K }\end{array}$ & $\begin{array}{c}\text { Freq., } \\
\text { GHz }\end{array}$ & $\begin{array}{c}\text { Performance } \\
\text { parameter }\end{array}$ & $\begin{array}{c}\text { Type A } \\
\left(10^{18} / \mathrm{cm}^{3}\right)\end{array}$ & $\begin{array}{c}\text { Type B } \\
\left(2 \times 10^{18} / \mathrm{cm}^{3}\right)\end{array}$ \\
\hline 300 & & $\mathrm{~g}_{\mathrm{m}}$ (mS/mm) & 380 & 450 \\
300 & 8 & Noise figure (dB) & 0.4 & - \\
300 & 8 & Associated gain (dB) & 15.2 & - \\
300 & 18 & Noise figure (dB) & 0.7 & 0.7 \\
300 & 18 & Assoc. gain (dB) & 11.5 & 15.0 \\
300 & 32 & Noise figure (dB) & 1.3 & 1.2 \\
300 & 32 & Assoc. gain (dB) & 7.5 & 10.0 \\
13 & 8.5 & Noise temp. (K) & 5.3 & 13.1 \\
13 & 8.5 & Assoc. gain (dB) & 13.9 & 14.5 \\
\hline
\end{tabular}



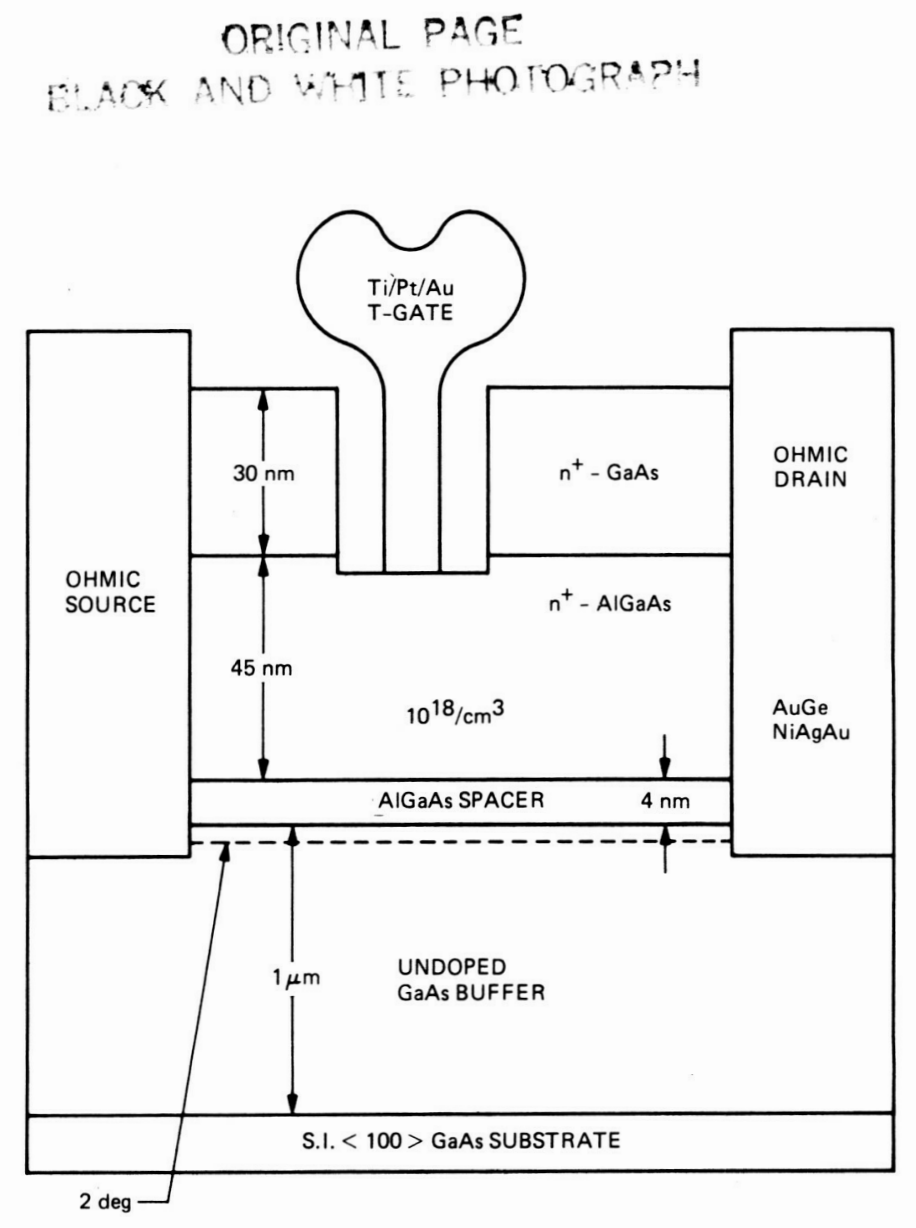

Fig. 1. Cross section of the $0.25-\mu \mathrm{m}$ T-gate HEMT.

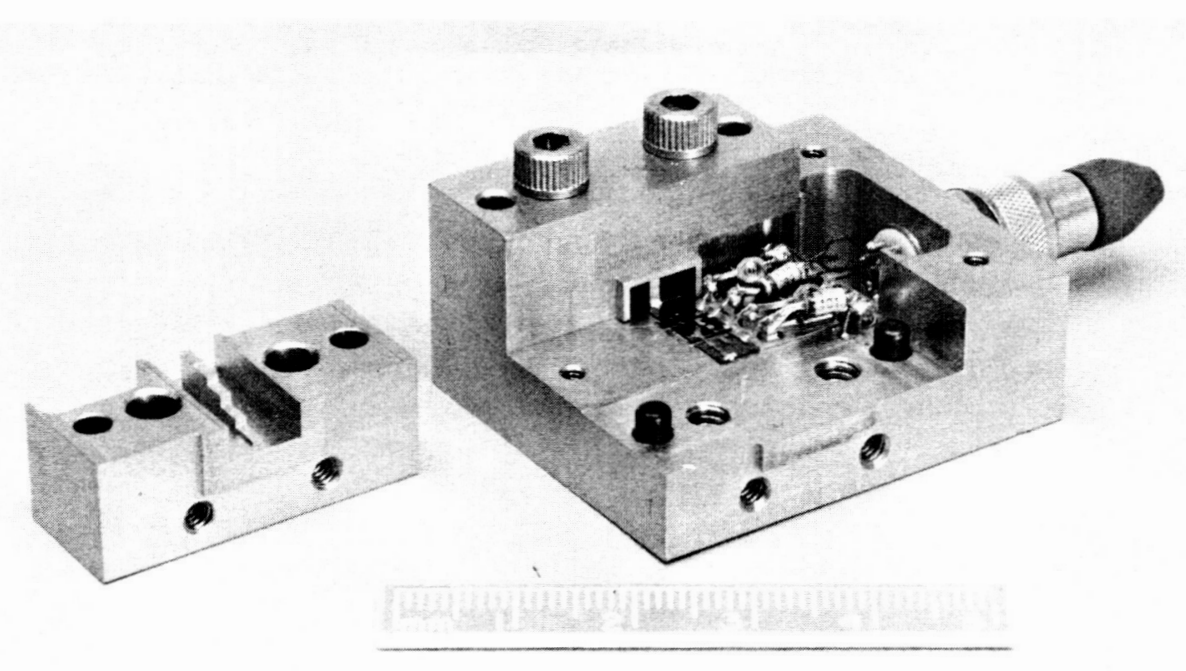

Fig. 2. Ka-band two-stage HEMT LNA. 

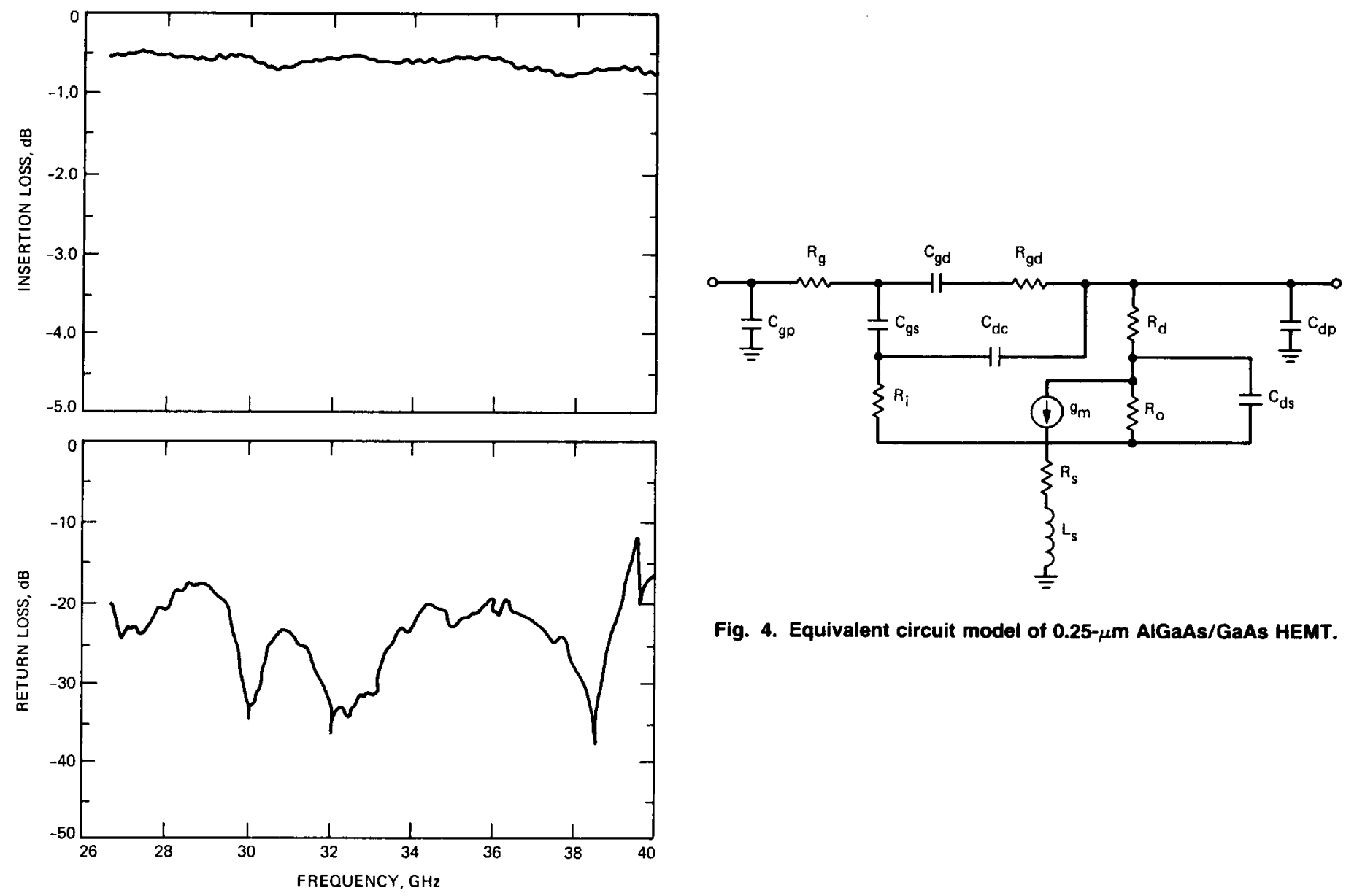

Fig. 4. Equivalent circuit model of $0.25-\mu \mathrm{m}$ AlGaAs/GaAs HEMT.

Fig. 3. Measured pertormance of Ka-band stepped ridge fixture.

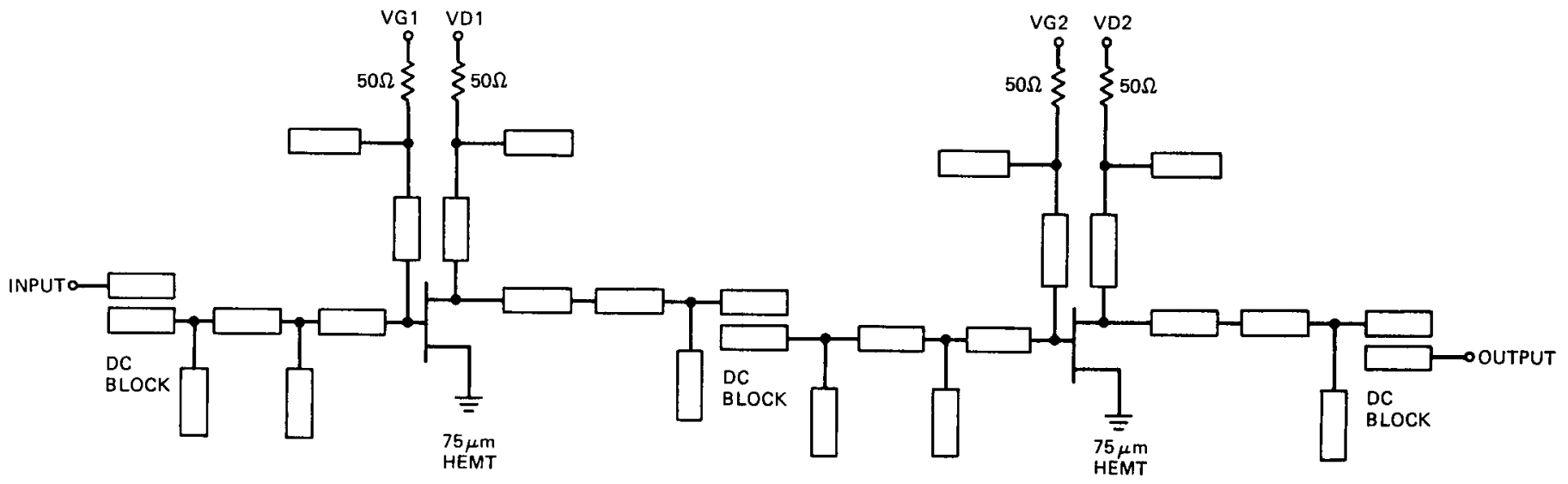

Fig. 5. Schematic diagram of two-stage hybrid LNA using $0.25 \times 75-\mu \mathrm{m}$ HEMTs. 


\section{ORIGINAL PAGE IS \\ OF POOR QUALITY}
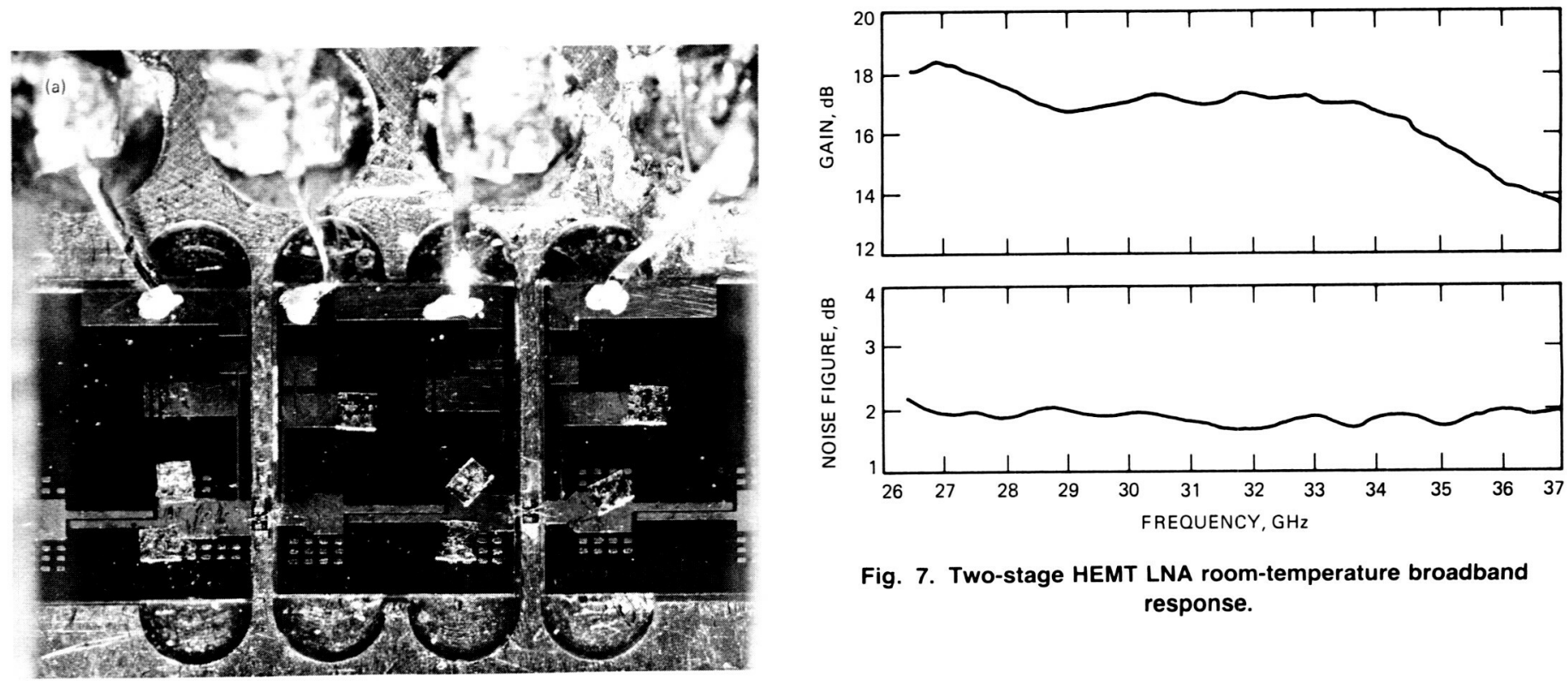

Fig. 7. Two-stage HEMT LNA room-temperature broadband response.

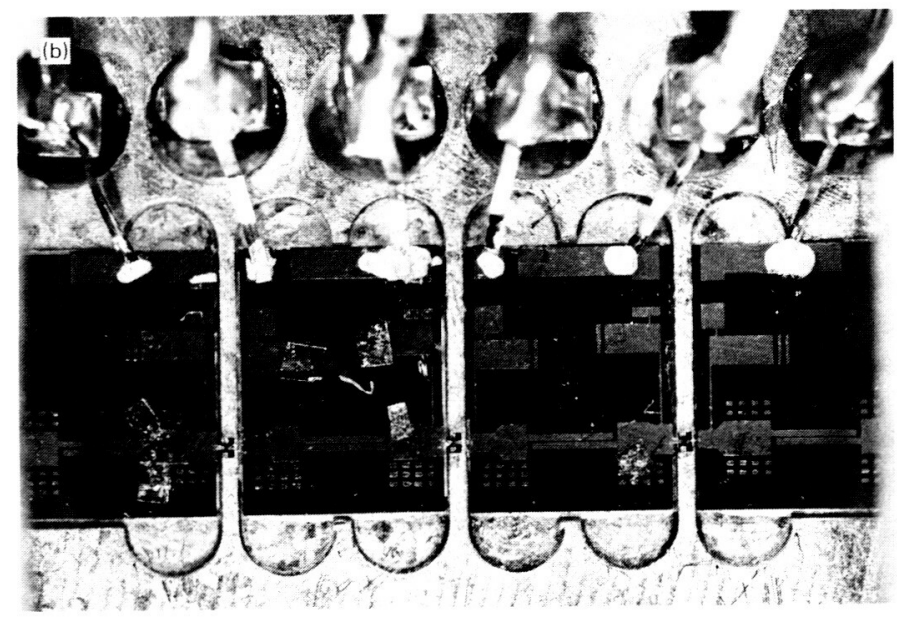

Fig. 6. Two-stage (a) and three-stage (b) HEMT LNA microstrip matching circuits.
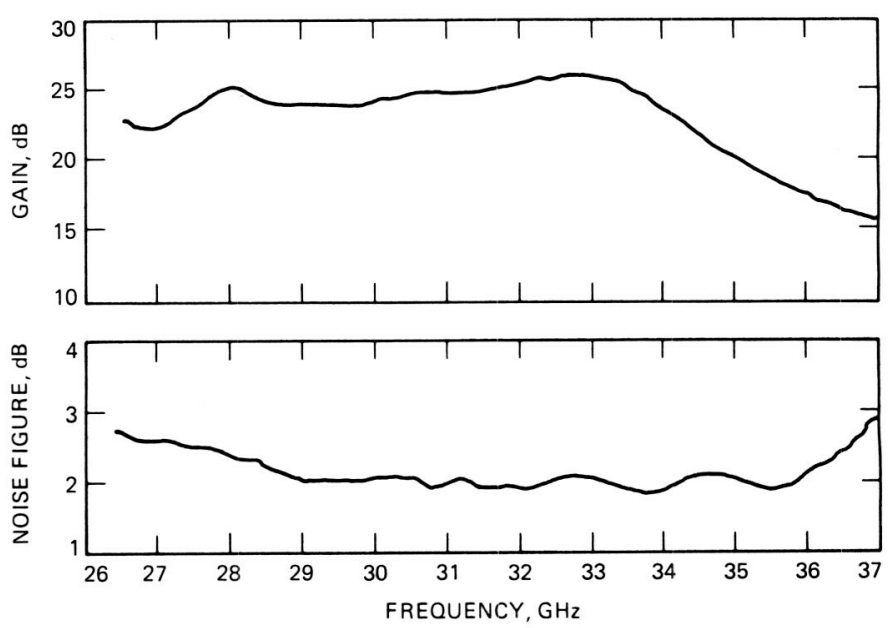

Fig. 8. Three-stage HEMT LNA room-temperature broadband response. 


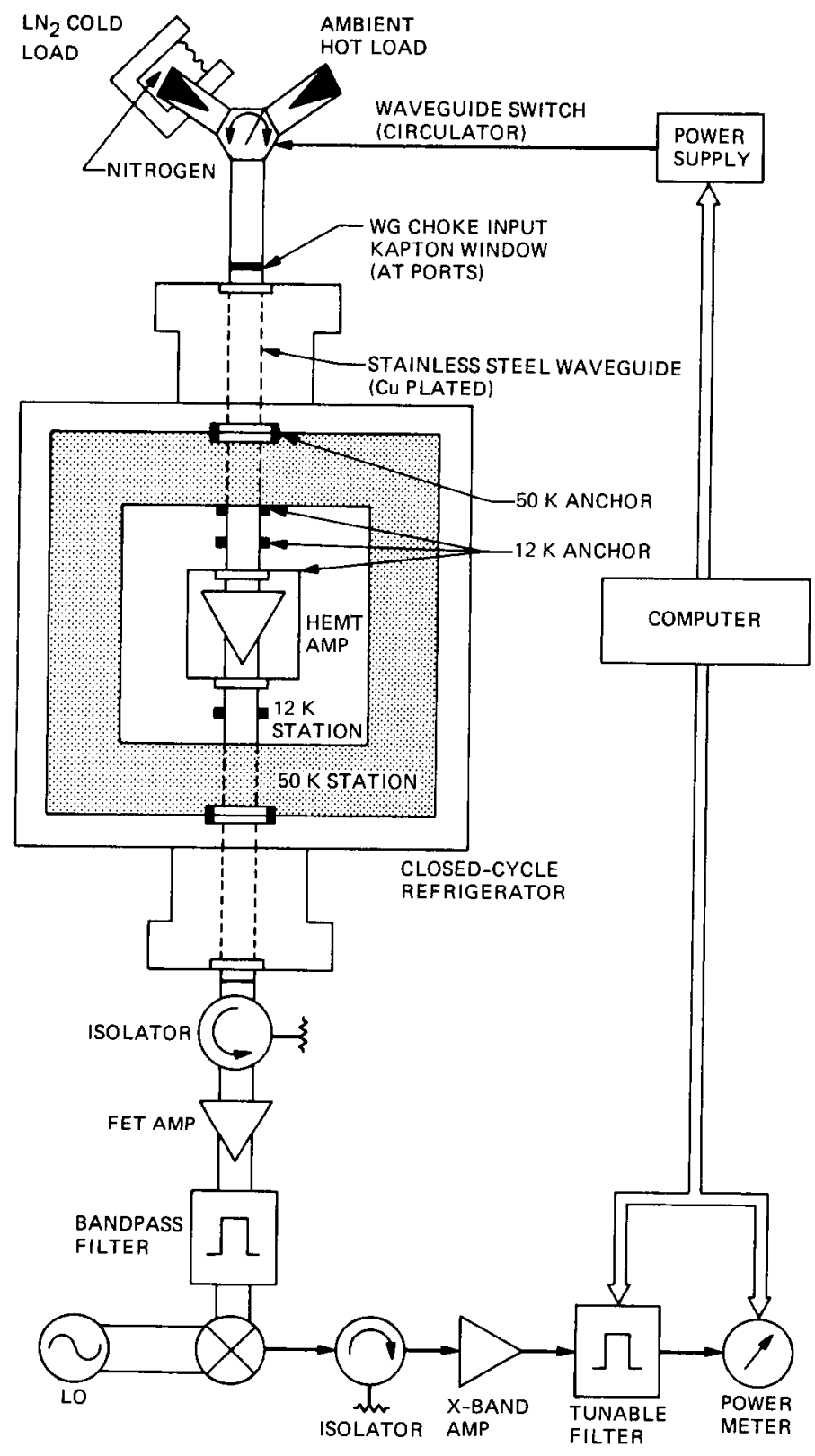

Fig. 9. 32-GHz cryogenic noise temperature measurement system. 


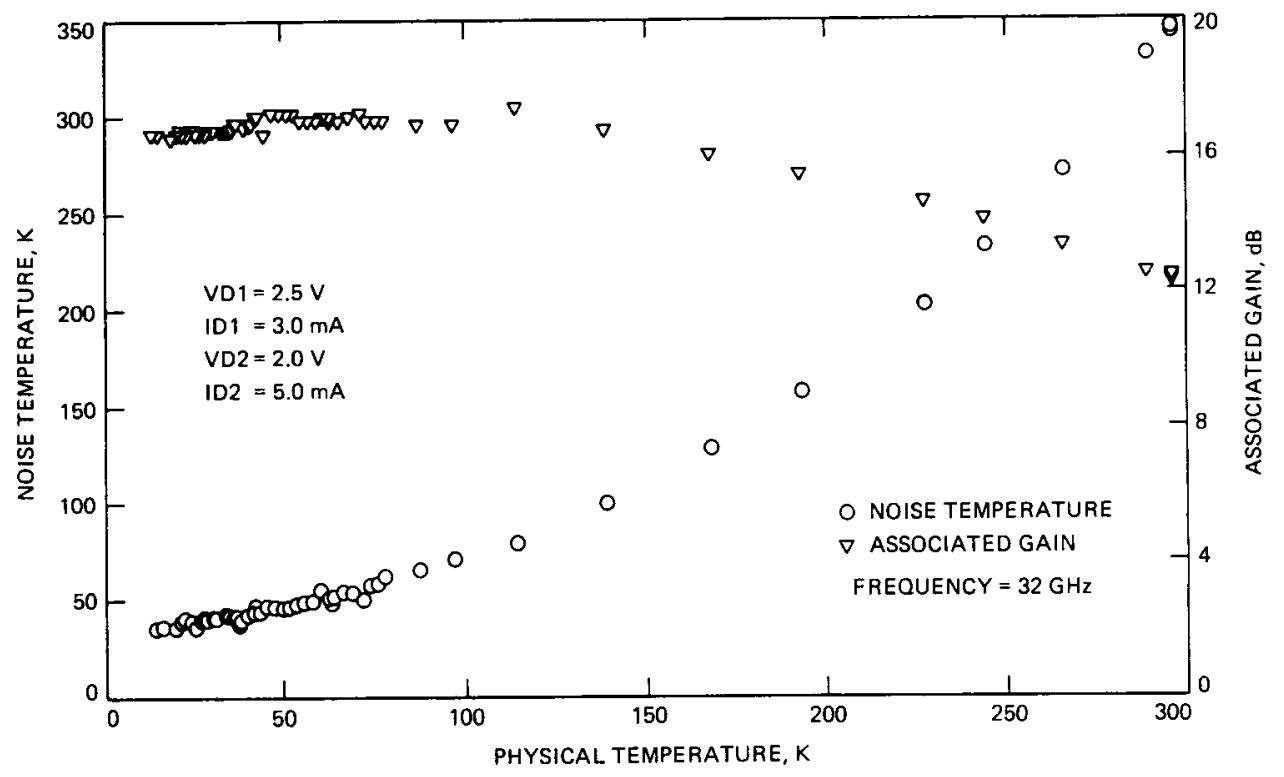

Fig. 10. Two-stage HEMT LNA noise temperature and gain cooling curve.

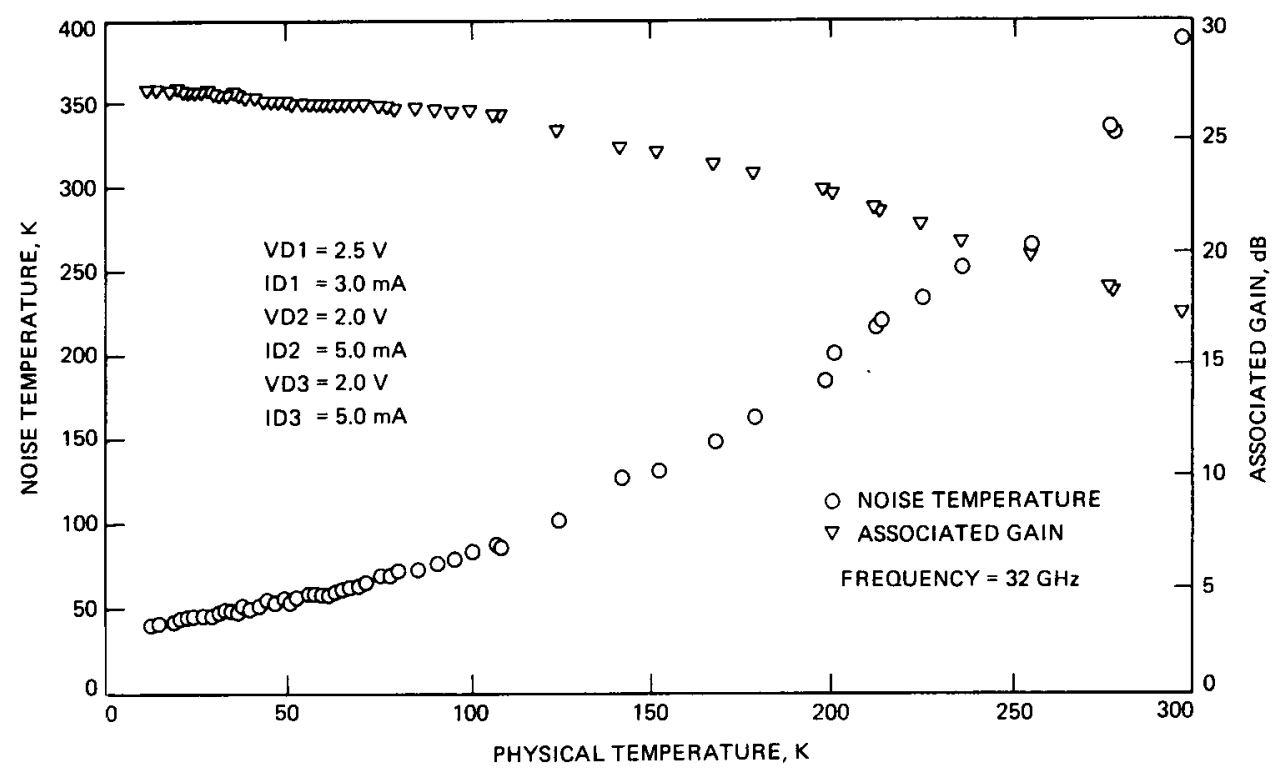

Fig. 11. Three-stage HEMT LNA noise temperature and gain cooling curve. 


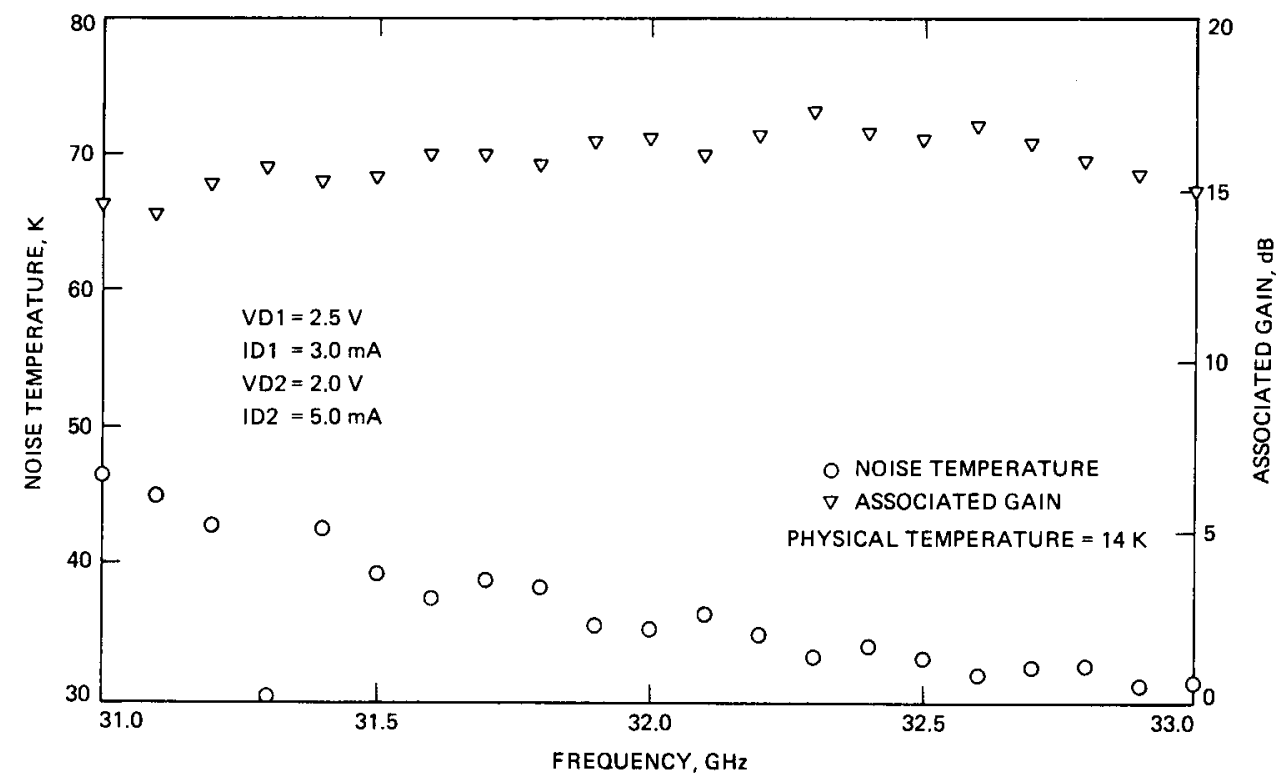

Fig. 12. Two-stage HEMT LNA at 14-K noise temperature and gain.

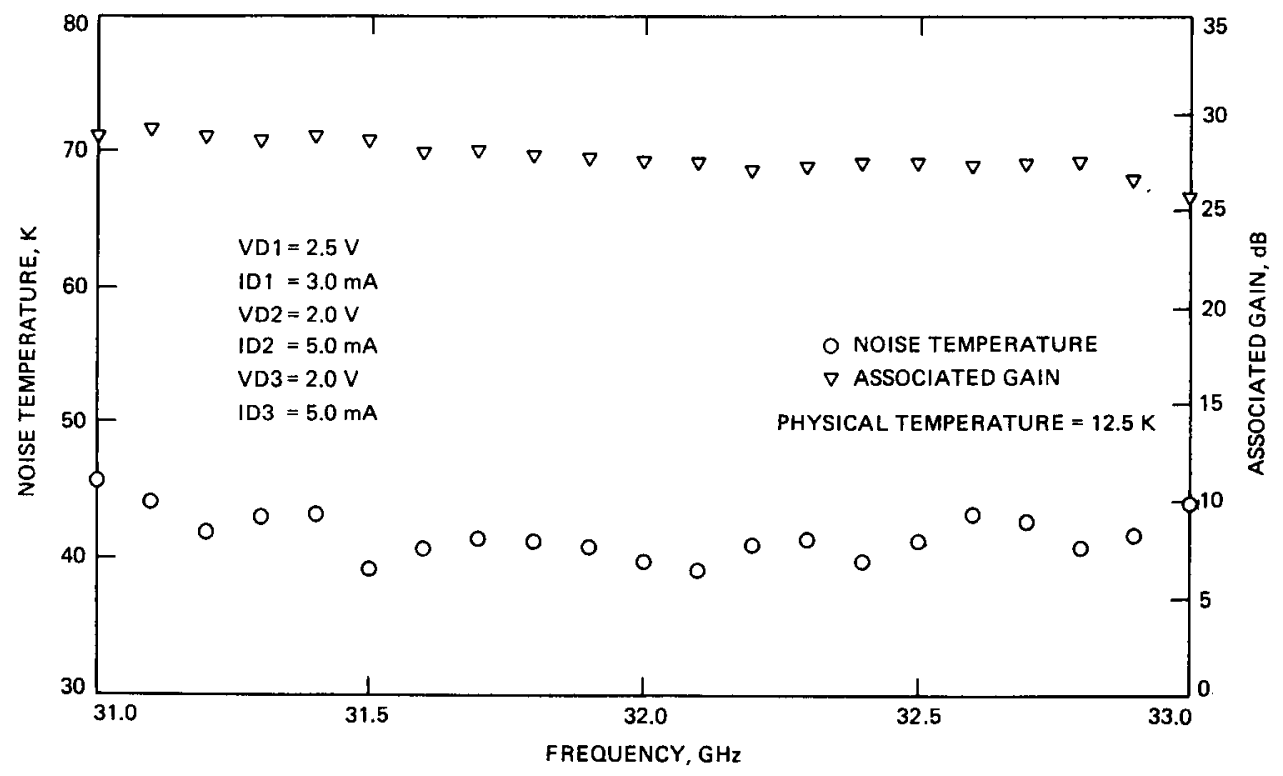

Fig. 13. Three-stage HEMT LNA at 12.5-K noise temperature and gain. 